\title{
A Cell Factory of a Fungicolous Fungus Calcarisporium arbuscula for Efficient Production of
}

\section{Natural Products}

Jin-Tao Cheng ${ }^{1,6}$, Jia-Hui Yu², Chen-Fan Sun ${ }^{1,6}$, Fei Cao ${ }^{1,6}$, You-Min Ying ${ }^{3}$, Zha-Jun Zhan ${ }^{3}$, Wen-Ju

Li $^{5}$, Xin-Ai Chen ${ }^{1,6}$, Qing-Wei Zhao ${ }^{1}$, Yong-Quan Li ${ }^{1,6 *}$, Li-She Gan ${ }^{2,4 *}$, Xu-Ming Mao ${ }^{1,6 *}$

${ }^{1}$ Institute of Pharmaceutical Biotechnology \& Research Center for Clinical Pharmacy, The First Affiliated Hospital, School of Medicine, Zhejiang University, Hangzhou 310058, China

${ }^{2}$ School of Biotechnology and Health Sciences, Wuyi University, Jiangmen, Guangdong 529020, People's Republic of China

${ }^{3}$ College of Pharmaceutical Science, Zhejiang University of Technology, Hangzhou 310014, China

${ }^{4}$ College of Pharmaceutical Sciences, Zhejiang University, Hangzhou 310058, People's Republic of China

${ }^{5}$ Jinan Samuel Pharmaceutical Co., Ltd of Shandong Province, Jinan 250100, China

${ }^{6}$ Zhejiang Provincial Key Laboratory for Microbial Biochemistry and Metabolic Engineering, Hangzhou 310058, China.

*To whom correspondence should be addressed:

Xu-Ming Mao, E-mail: xmmao@zju.edu.cn; Tel: 86-571-88981335.

Li-She Gan, E-mail: 1sgan@zju.edu.cn; Tel: 86-571-88208457.

Yong-Quan Li, E-mail: lyq@zju.edu.cn; Tel: 86-571-88206632. 


\section{Table of contents}

\section{Supplementary Tables:}

Table S1. Annotation of the selected 14 promoter regions

Table S2. Annotation of the selected three housekeeping genes and PKS51 S4

Table S3. Strains and plasmids used in this work $\quad$ S5

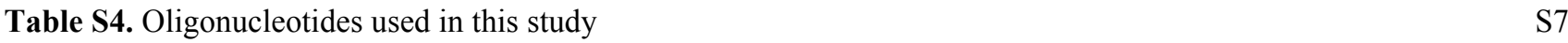

Table S5. NMR data of the isolated compound $1 \quad$ S8

Table S6. Energy (298.15 K) analysis for $\left(1^{\prime} S, 3^{\prime} R\right)-1$ and $\left(1^{\prime} S, 3^{\prime} S\right)$-1 $\quad$ S9

Table S7. Calculated 1H-NMR chemical shifts for conformers of (1' $\left.S, 3^{\prime} R\right)-1$ and $\left(1^{\prime} S, 3^{\prime} S\right)-1 \quad$ S10

Table S8. Calculated 13C-NMR chemical shifts for conformers of $\left(1^{\prime} S, 3^{\prime} R\right)-1$ and $\left(1^{\prime} S, 3^{\prime} S\right)-1 \quad$ S11

Table S9. DP4+ evaluation of theoretical and experimental data $\quad$ S13

Table S10. Calculated ECD Data for (1'S,3'S)-1 in gas phase $\quad$ S14

\section{Supplementary Figures:}

Figure S1. The directional insertion of the promoters $\quad$ S18

Figure S2. Expression of the red fluorescent protein (RFP) under 14 different promoters in $\triangle a u r A . \quad$ S19

$\begin{array}{ll}\text { Figure S3. Metabolite profile analysis based on iso-absorbance plot } & \text { S20 }\end{array}$

Figure S4. Metabolite profile analysis and HPLC analysis of three different hosts $\quad$ S21

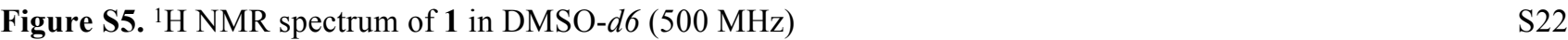

Figure S6. ${ }^{13} \mathrm{C}$ NMR spectrum of $\mathbf{1}$ in DMSO- $d 6 \quad$ S23

Figure S7. The HSQC spectrum of $\mathbf{1}$ in DMSO- $d 6 \quad$ S24

Figure S8. The HMBC spectrum of $\mathbf{1}$ in DMSO- $d 6 \quad$ S25

Figure S9. The COSY spectrum of $\mathbf{1}$ in DMSO- $d 6 \quad$ S26

$\begin{array}{ll}\text { Figure S10. The NOESY spectrum of } \mathbf{1} \text { in DMSO- } d 6 & \text { S27 }\end{array}$

Figure S11. Determination of the structure of compound $1 \quad$ S28

$\begin{array}{lr}\text { Figure S12. Determination of relative and absolute configuration of } \mathbf{1} & \text { S29 }\end{array}$

Figure S13. B3LYP/6-31G(d) optimized lowest energy conformers for $\left(1^{\prime} S, 3^{\prime} R\right)-\mathbf{1} \quad S 30$

Figure S14. B3LYP/6-31G(d) optimized lowest energy conformers for (1'S,3'S) -1 $\quad$ S31

Figure S15. LC-MS analysis in positive ion mode and UV analysis of compound $\mathbf{2}$ and compound $3 \quad$ S32

Figure S16. LC-MS analysis and UV analysis of compound $4 \quad$ S33 
Table S1. Annotation of the selected 14 promoter regions from C. aubuscula based on the RNA-Seq data.

\begin{tabular}{|l|l|l|c|c|c|c|c|}
\hline Number & $\begin{array}{l}\text { Gene } \\
\text { ID }\end{array}$ & $\begin{array}{l}\text { Promoter } \\
\text { name }\end{array}$ & $\begin{array}{c}\text { WT1 } \\
(\text { PFKM })\end{array}$ & $\begin{array}{c}\text { WT2 } \\
(\text { PFKM })\end{array}$ & $\begin{array}{c}\text { WT2 } \\
(\text { PFKM })\end{array}$ & SD & $\begin{array}{c}\text { Average value } \\
\text { (PFKM) }\end{array}$ \\
\hline P1 & $A 01884$ & $A 01881 p$ & 13359 & 12498 & 13966 & 737 & 13274 \\
\hline P2 & $A 02690$ & $A 02690 p$ & 4897 & 5432 & 5354 & 289 & 5228 \\
\hline P3 & $A 08158$ & $A 08158 p$ & 4230 & 3765 & 3917 & 237 & 3971 \\
\hline P4 & $A 03528$ & $A 03528 p$ & 3454 & 3960 & 4170 & 368 & 3861 \\
\hline P5 & $A 03362$ & $A 03362 p$ & 3342 & 3789 & 4010 & 340 & 3714 \\
\hline P6 & $A 01996$ & $A 01996 p$ & 4052 & 3289 & 2803 & 629 & 3382 \\
\hline P7 & $A 02236$ & $A 02236 p$ & 3568 & 3125 & 3082 & 269 & 3259 \\
\hline P8 & $A 05995$ & $A 05995 p$ & 2893 & 3124 & 3183 & 153 & 3067 \\
\hline P9 & $A 00271$ & $A 00271 p$ & 2789 & 2901 & 3474 & 367 & 3055 \\
\hline P10 & $A 04406$ & $A 04406 p$ & 3123 & 2798 & 2590 & 268 & 2837 \\
\hline P11 & $A 08989$ & $A 08989 p$ & 2897 & 2589 & 2719 & 154 & 2735 \\
\hline P12 & $A 01394$ & $A 01394 p$ & 2987 & 2154 & 2256 & 454 & 2466 \\
\hline P13 & $A 04086$ & $A 04086 p$ & 2145 & 2298 & 2511 & 183 & 2318 \\
\hline P14 & $A 05840$ & $A 05840 p$ & 2437 & 2387 & 1895 & 299 & 2240 \\
\hline
\end{tabular}


Table S2. Annotation of the selected three housekeeping genes and PKS51 from C. aubuscula based on the RNASeq data.

\begin{tabular}{|l|l|c|c|c|c|}
\hline $\begin{array}{l}\text { Gene } \\
\text { ID }\end{array}$ & Description & $\begin{array}{c}\text { WT1 } \\
(\text { PFKM })\end{array}$ & $\begin{array}{c}\text { WT2 } \\
(\text { PFKM })\end{array}$ & $\begin{array}{c}\text { WT2 } \\
\text { (PFKM } \\
\text { A07909 }\end{array}$ & Average value (PFKM) \\
\hline Putative actin protein & 578 & 678 & 724 & 660 \\
\hline$A 08632$ & Beta-tubulin & 513 & 490 & 440 & 481 \\
\hline$A 01295$ & $\begin{array}{l}\text { Glyceraldehyde-3- } \\
\text { phosphate } \\
\text { dehydrogenase }\end{array}$ & 986 & 938 & 947 & 957 \\
\hline Polyketide synthase & \begin{tabular}{l} 
(PKS51) \\
\hline
\end{tabular} & 0.2 & 0.5 & 0.2 & 0.3 \\
\hline
\end{tabular}


Table S3. Strains and plasmids used in this work

\begin{tabular}{|c|c|c|}
\hline Strains/Plasmid & Description & Source \\
\hline C. arbuscula NRRL 3705 & Wild strain & Our lab \\
\hline C. arbuscula $\triangle$ aurA & $\begin{array}{l}\text { deficient of aurA (encoding aurovertin polyketide } \\
\text { synthase) }\end{array}$ & Our lab \\
\hline $\begin{array}{l}\text { M. anisopliae ARSEF } \\
549\end{array}$ & contain a large number of gene clusters & \\
\hline M. robertsii ARSEF & contain a large number of gene clusters & \\
\hline 2575 & & \\
\hline A. nidulans LO8030 & an ideal cell factory & \\
\hline A. oryzae NSAR1 & an ideal cell factory & \\
\hline \multicolumn{3}{|l|}{ Plasmids } \\
\hline pFGL-neoR & $n e o R$ cloned in NcoI-digested pFGL-ANgpdAp & Our lab \\
\hline pFGL-neoR-RFP & $\begin{array}{l}\text { pFGL-neoR derived, carrying a } r f p \text { gene from } \\
\text { pFGL-tubCp-RFP at BamHI }\end{array}$ & This study \\
\hline pFGL-neoR-RFP-L-R & $\begin{array}{l}\text { pFGL-neoR-RFP derived, the upstream and } \\
\text { downstream homologous regions of aurA }\end{array}$ & \\
\hline pFGL-P1-RFP-L-R & $\begin{array}{l}\text { pFGL-neoR-RFP-L-R derived, carrying \#1 } \\
\text { promoter at BamHI }\end{array}$ & This study \\
\hline pFGL-P2-RFP-L-R & $\begin{array}{l}\text { pFGL-neoR-RFP-L-R derived, carrying \#2 } \\
\text { promoter at BamHI }\end{array}$ & This study \\
\hline pFGL-P3-RFP-L-R & $\begin{array}{l}\text { pFGL-neoR-RFP-L-R derived, carrying \#3 } \\
\text { promoter at BamHI }\end{array}$ & This study \\
\hline pFGL-P4-RFP-L-R & $\begin{array}{l}\text { pFGL-neoR-RFP-L-R derived, carrying \#4 } \\
\text { promoter at BamHI }\end{array}$ & This study \\
\hline pFGL-P5-RFP-L-R & $\begin{array}{l}\text { pFGL-neoR-RFP-L-R derived, carrying \#5 } \\
\text { promoter at BamHI }\end{array}$ & This study \\
\hline pFGL-P6-RFP-L-R & $\begin{array}{l}\text { pFGL-neoR-RFP-L-R derived, carrying \#6 } \\
\text { promoter at BamHI }\end{array}$ & This study \\
\hline pFGL-P7-RFP-L-R & $\begin{array}{l}\text { pFGL-neoR-RFP-L-R derived, carrying \#7 } \\
\text { promoter at BamHI }\end{array}$ & This study \\
\hline pFGL-P8-RFP-L-R & $\begin{array}{l}\text { pFGL-neoR-RFP-L-R derived, carrying \#8 } \\
\text { promoter at } B a m \mathrm{HI}\end{array}$ & This study \\
\hline pFGL-P9-RFP-L-R & $\begin{array}{l}\text { pFGL-neoR-RFP-L-R derived, carrying \#9 } \\
\text { promoter at BamHI }\end{array}$ & This study \\
\hline pFGL-P10-RFP-L-R & $\begin{array}{l}\text { pFGL-neoR-RFP-L-R derived, carrying } \\
\# 10 \text { promoter at BamHI }\end{array}$ & This study \\
\hline pFGL-P11-RFP-L-R & $\begin{array}{l}\text { pFGL-neoR-RFP-L-R derived, carrying \#11 } \\
\text { promoter at BamHI }\end{array}$ & This study \\
\hline pFGL-P12-RFP-L-R & $\begin{array}{l}\text { pFGL-neoR-RFP-L-R derived, carrying \#12 } \\
\text { promoter at BamHI }\end{array}$ & This study \\
\hline pFGL-P13-RFP-L-R & $\begin{array}{l}\text { pFGL-neoR-RFP-L-R derived, carrying \#13 } \\
\text { promoter at } B a m \mathrm{HI}\end{array}$ & This study \\
\hline
\end{tabular}




\begin{tabular}{lll} 
pFGL-P14-RFP-L-R & $\begin{array}{l}\text { pFGL-neoR-RFP-L-R derived, carrying \#14 } \\
\text { promoter at BamHI }\end{array}$ & This study \\
pFGL-tubCp-RFP-L-R & $\begin{array}{l}\text { pFGL-neoR-RFP-L-R derived, carrying tubCp } \\
\text { promoter at BamHI }\end{array}$ & This study \\
pFGL-aurBp & pFGL-neoR derived, carrying \#8 promoter BamHI & This study \\
pFGL-aurBp-TF51 & pFGL- aurBp derived, carrying TF51 at BamHI & This study \\
pFGL-aurBp-PKS51 & pFGL- aurBp derived, carrying TFPKS at BamHI & This study \\
pFGL-aurBp-MavA & pFGL- aurBp derived, carrying mavA at BamHI & This study \\
pFGL-aurBp-MrvA & pFGL- aurBp derived, carrying mrvA at BamHI & This study \\
pFGL-aurBp-MrPKS1 & pFGL- aurBp derived, carrying mrpks 1 at BamHI & This study \\
\hline
\end{tabular}


Table S4. Oligonucleotides used in this study

\begin{tabular}{|c|c|}
\hline RFP-F & GAGGTAATCCTCCCGGGAACTGTGGACGATCTCGTC \\
\hline RFP-R & CTCTAGAACTAGTGGATCCATGGTGAGCAAGGGCGAG \\
\hline P1-F & CTCCTCGCCCTTGCTCACCATATTGATGAGGGTGTTGGAAGTGTG \\
\hline P1-R & GACCGACCACCTAACAACGGATCCCACCATGTGTAACGAGGACTG \\
\hline $\mathrm{P} 2-\mathrm{F}$ & СTCCTCGCCCTTGCTCACCATGATGGGCAACTTTTACGGATCG \\
\hline $\mathrm{P} 2-\mathrm{R}$ & GTTGACCGACCACCTAACAACGGATCCGGAAAGGAGCGAGAACGATG \\
\hline P3-F & CCTCGCCCTTGCTCACCATTTTGTTCTGATGTGATTGTCGTC \\
\hline $\mathrm{P} 3-\mathrm{R}$ & GACCGACCACCTAACAACGGATCCGTTGGCTGTTGCAGTCGAG \\
\hline P4-F & CCTCCTCGCCCTTGCTCACCATTTTGACGGTATTAAAAGGTTACTCG \\
\hline P4-R & CGACCACCTAACAACGGATCCTTTGCAAGCCGTGATTTCCG \\
\hline P5-F & СCTCCTCGCCCTTGCTCACCATGTTGACTGATGTTCAAAAAATAATTCTTG \\
\hline P5-R & GACCGACCACCTAACAACGGATCCGGTGTTTACGTAAGGCTCTG \\
\hline P6-F & СТCCTCGCCCTTGCTCACCATCTTGACGGTGGTTGTTGGGG \\
\hline P6-R & GTTGACCGACCACCTAACAACGGATCCTTTGTTGCGAAGAGTGTGCTG \\
\hline P7-F & СCTCCTCGCCCTTGCTCACCATGTTGGCGGTATTTTCAATGGAC \\
\hline P7-R & GTTGACCGACCACCTAACAACGGATCCGGTGGAGGGTGGGAATG \\
\hline P8-F & СTCCTCGCCCTTGCTCACCATGATGGGTATTGAGTAGGTAAGGC \\
\hline P8-R & CGCCTTGGTCTGACCAGCGGATCCGTTGTTAGGTGGTCGGTC \\
\hline P9-F & CTCCTCGCCCTTGCTCACCATGGCACTGGCAAGATGTGTTTG \\
\hline P9-R & GTTGACCGACCACCTAACAACGGATCCGAAGTGGTAGAGACTCAGGC \\
\hline P10-F & CCTCGCCCTTGCTCACCATCTTGGCGGTATCTGAGGC \\
\hline P10-R & CCGACCACCTAACAACGGATCCCTTGCCTAACTGTGGAGG \\
\hline P11-F & CCTCGCCCTTGCTCACCATCTTGCCTAACTGTGGAGG \\
\hline P11-R & GTTGACCGACCACCTAACAACGGATCCCTTGGCGGTATCTGAGGC \\
\hline P12-F & CCTCGCCCTTGCTCACCATTGACGGCTTGAAATTCTGATG \\
\hline P12-R & GTTGACCGACCACCTAACAACGGATCCTCGCATTCTATTCGTTCTGTGC \\
\hline P13-F & CCTCCTCGCCCTTGCTCACCATTTTTGTGAGTTCAGGTGAATCG \\
\hline P13-R & GACCGACCACCTAACAACGGATCCTGTGGATGTTAGGGGTTGG \\
\hline P14-F & CCTCGCCCTTGCTCACCATTTTTGCGGTTGTGAGTGTTTG \\
\hline P14-R & GTTGACCGACCACCTAACAACGGATCCAGGGAAGCTGAAGATATATGACG \\
\hline aurBp-F & GTCATCCTTGTAATCGGATCCAAGCTTGATGGGTATTGAGTAGGTAAGG \\
\hline aurBp-R & CAGGTCGACTCTAGAACTAGTGCTGGTCAGACCAAGGCG \\
\hline $51 \mathrm{TF}-\mathrm{F}$ & CGTCGTCATCCTTGTAATCGGATCCTTACTCACCGGCTGTGGGGAC \\
\hline $51 \mathrm{TF}-\mathrm{R}$ & CCTACTCAATACCCATCAAGCTTATGCCCCCCAGCTCTGTC \\
\hline $51 \mathrm{PKS}-\mathrm{F}$ & CTTATCGTCGTCATCCTTGTAATCTCAACAATCATCTTCCTCTCCAATCAG \\
\hline 51PKS-R & СCTACTCAATACCCATCAAGCTTATGGGTGAGTACCACGCCCAATGC \\
\hline MavA-F & CGTCGTCATCCTTGTAATCGTCTTTTCCTACATTTGGAATGAAGC \\
\hline MavA-R & GCCTTACCTACTCAATACCCATCAAGCTTATGACACCAGAGCCAATAGCC \\
\hline MrvA-F & CACTTATCGTCGTCATCCTTGTAATCGTCTTTTCCTACATTTGGAATGAAGC \\
\hline MrvA-R & CTTACCTACTCAATACCCATCAAGCTTATGACACCAGAGCCAATAGC \\
\hline MrPKS1-F & CACTTATCGTCGTCATCCTTGTAATCACCAAACGTACTCCGAATAAAAGC \\
\hline MrPKS1-R & CTTACCTACTCAATACCCATCAAGCTTATGAACCACGTGACGATCAAG \\
\hline
\end{tabular}


Table S5. NMR data of the isolated compound $\mathbf{1}$

\begin{tabular}{lll}
\hline No. & $\delta_{\mathrm{H}}$ & $\delta_{\mathrm{C}}$ \\
\hline 1 & - & $163.7^{\mathrm{a}}$ \\
2 & - & 120.3 \\
3 & - & $163.5^{\mathrm{a}}$ \\
4 & $7.05(\mathrm{~s})$ & $108.7^{\mathrm{b}}$ \\
$4 \mathrm{a}$ & - & 132.5 \\
5 & $7.04(\mathrm{~d}, 2.4)$ & $108.6^{\mathrm{b}}$ \\
6 & - & $164.2^{\mathrm{a}}$ \\
7 & $6.52(\mathrm{~d}, 2.4)$ & 108.1 \\
8 & - & 165.0 \\
$8 \mathrm{a}$ & - & $109.7^{\mathrm{c}}$ \\
9 & - & 188.7 \\
$9 \mathrm{a}$ & - & $109.7^{\mathrm{c}}$ \\
10 & - & 181.1 \\
$10 \mathrm{a}$ & - & 134.9 \\
$1^{\prime}$ & $3.67(\mathrm{~m})$ & 40.9 \\
$2^{\prime}$ & $3.82(\mathrm{dd}, 9.8,8.2)$ & 59.8 \\
$3^{\prime}$ & $3.58(\mathrm{~m})$ & 70.7 \\
$4^{\prime}$ & $4.18(\mathrm{~m})$ & 64.6 \\
\hline
\end{tabular}


Table S6. Energy (298.15 K) analysis for (1'S,3'R) -1 and (1'S,3'S) -1

\begin{tabular}{|l|l|l|l|}
\hline Conf. & G (Hartree $)$ & \multicolumn{4}{|c|}{$\Delta \mathrm{G}(\mathrm{Kcal} / \mathrm{mol})$} & \multicolumn{2}{|c|}{ Boltzmann Distribution } \\
\hline & \multicolumn{3}{|c|}{$\left(1^{\prime} S, 3^{\prime} R\right)-1$} \\
\hline C1 & -1372.292112 & 1.92833823 & 0.014101218 \\
\hline C2 & -1372.294813 & 0.23343372 & 0.246753995 \\
\hline C3 & -1372.295185 & 0 & 0.365981898 \\
\hline C4 & -1372.294345 & 0.5271084 & 0.150275893 \\
\hline C5 & -1372.294717 & 0.29367468 & 0.222886996 \\
\hline & \multicolumn{3}{|l|}{$\left(1^{\prime} S, 3^{\prime} S\right)-1$} \\
\hline C1 & -1372.296978 & 0.28112448 & 0.332524246 \\
\hline C2 & -1372.297426 & 0 & 0.534557143 \\
\hline C3 & -1372.295229 & 1.37863947 & 0.052110922 \\
\hline C4 & -1372.295643 & 1.11885033 & 0.080807688 \\
\hline
\end{tabular}


Table S7. Calculated ${ }^{1} \mathrm{H}-\mathrm{NMR}$ chemical shifts for conformers of $\left(1^{\prime} S, 3^{\prime} R\right)-\mathbf{1}$ and $\left(1^{\prime} S, 3^{\prime} S\right)-\mathbf{1}$.

\begin{tabular}{|l|l|l|l|l|l|}
\hline No. & \multicolumn{5}{|c|}{$\left(1^{\prime} S, 3^{\prime} R\right)-1$} \\
\hline & C1 & C2 & C3 & C4 & C5 \\
\hline 4 & 7.7264 & 7.8427 & 7.8090 & 7.8464 & 7.8129 \\
\hline 5 & 7.1716 & 7.7282 & 7.1910 & 7.7328 & 7.1953 \\
\hline 7 & 6.9620 & 6.3980 & 6.9781 & 6.4040 & 6.9835 \\
\hline 1, & 4.0519 & 3.6853 & 3.6890 & 3.7393 & 3.7421 \\
\hline 2, & 4.5173 & 4.2735 & 4.2742 & 4.5730 & 4.5725 \\
\hline & 3.7531 & 4.1363 & 4.1342 & 4.0590 & 4.0569 \\
\hline 3, & 4.3549 & 4.6757 & 4.6751 & 4.2534 & 4.2540 \\
\hline 4, & 3.2340 & 3.4340 & 3.4287 & 3.0005 & 2.9958 \\
\hline & 3.8268 & 3.4726 & 3.4736 & 3.5875 & 3.5912 \\
\hline
\end{tabular}

\begin{tabular}{|l|l|l|l|l|}
\hline No. & \multicolumn{3}{|c|}{$\left(1^{\prime} S, 3\right.$ 'S $)-1$} \\
\hline & C1 & C2 & C3 & C4 \\
\hline 4 & 7.7340 & 7.7003 & 7.8272 & 7.7939 \\
\hline 5 & 7.6974 & 7.1634 & 7.7222 & 7.1873 \\
\hline 7 & 6.3793 & 6.9567 & 6.3877 & 6.9650 \\
\hline 1, & 4.1128 & 4.1153 & 3.1725 & 3.1679 \\
\hline 2, & 3.5100 & 3.5064 & 4.1294 & 4.1282 \\
\hline & 4.5429 & 4.5345 & 3.9703 & 3.9685 \\
\hline 3, & 3.9870 & 3.9833 & 4.1175 & 4.1188 \\
\hline 4, & 3.7819 & 3.7819 & 4.0670 & 4.0671 \\
\hline & 3.8665 & 3.8677 & 3.2564 & 3.2487 \\
\hline
\end{tabular}


Table S8. Calculated ${ }^{13} \mathrm{C}-\mathrm{NMR}$ chemical shifts for conformers of (1'S, $\left.3^{\prime} R\right)-\mathbf{1}$ and (1'S, $\left.3^{\prime} S\right)-\mathbf{1}$

\begin{tabular}{|c|c|c|c|c|c|}
\hline \multirow[t]{2}{*}{ No. } & \multicolumn{5}{|c|}{$\left(1^{\prime} S, 3^{\prime} R\right)-1$} \\
\hline & $\mathrm{C} 1$ & $\mathrm{C} 2$ & $\mathrm{C} 3$ & $\mathrm{C} 4$ & $\mathrm{C} 5$ \\
\hline 1 & 162.896 & 160.5123 & 160.5434 & 160.4442 & 160.48 \\
\hline 2 & 114.9013 & 120.2623 & 120.5226 & 120.1835 & 120.4601 \\
\hline 3 & 161.6698 & 160.7648 & 160.6796 & 160.9901 & 160.9098 \\
\hline 4 & 112.0497 & 112.561 & 112.5008 & 112.8715 & 112.8132 \\
\hline $4 a$ & 132.9655 & 132.977 & 132.7119 & 132.9792 & 132.7137 \\
\hline 5 & 105.3867 & 108.9431 & 105.7833 & 109.0247 & 105.8629 \\
\hline 6 & 159.5602 & 160.0028 & 159.8602 & 160.0534 & 159.9023 \\
\hline 7 & 107.1675 & 104.2684 & 107.2105 & 104.2802 & 107.2237 \\
\hline 8 & 163.7777 & 163.4952 & 163.9202 & 163.5196 & 163.9401 \\
\hline $8 a$ & 110.3344 & 109.8281 & 110.227 & 109.8111 & 110.2079 \\
\hline 9 & 185.5835 & 185.7505 & 185.9118 & 185.8375 & 185.995 \\
\hline $9 a$ & 107.7048 & 109.523 & 109.4846 & 109.4666 & 109.4284 \\
\hline 10 & 179.0517 & 178.5811 & 178.7428 & 178.5514 & 178.7155 \\
\hline $10 \mathrm{a}$ & 134.2765 & 134.8473 & 134.2126 & 134.8437 & 134.2138 \\
\hline 1 ' & 43.0167 & 40.1456 & 40.1563 & 39.9144 & 39.9245 \\
\hline 2 & 63.7864 & 74.4417 & 74.4372 & 71.3126 & 71.3072 \\
\hline 3, & 75.0354 & 81.7658 & 81.7726 & 84.6482 & 84.6561 \\
\hline 4 ' & 66.2363 & 66.0813 & 66.0679 & 66.6439 & 66.6389 \\
\hline
\end{tabular}




\begin{tabular}{|c|c|c|c|c|}
\hline No. & & & , $\left.3^{\prime} S\right)-1$ & \\
\hline & $\mathrm{C} 1$ & $\mathrm{C} 2$ & $\mathrm{C} 3$ & $\mathrm{C} 4$ \\
\hline 1 & 160.2276 & 160.2631 & 160.3152 & 160.3623 \\
\hline 2 & 118.2985 & 118.5607 & 119.2073 & 119.5081 \\
\hline 3 & 162.7676 & 162.6667 & 162.5149 & 162.4252 \\
\hline 4 & 112.9304 & 112.896 & 112.9126 & 112.8727 \\
\hline $4 a$ & 132.8241 & 132.5625 & 132.1851 & 131.917 \\
\hline 5 & 108.4607 & 105.3393 & 109.0318 & 105.8926 \\
\hline 6 & 159.6549 & 159.5197 & 160.0687 & 159.9346 \\
\hline 7 & 104.0632 & 106.9755 & 104.1362 & 107.0451 \\
\hline 8 & 163.2538 & 163.7121 & 163.4223 & 163.8557 \\
\hline $8 a$ & 110 & 110.3974 & 109.7371 & 110.1347 \\
\hline 9 & 185.4027 & 185.5423 & 185.8271 & 186.004 \\
\hline $9 \mathrm{a}$ & 108.1392 & 108.1175 & 108.8004 & 108.7754 \\
\hline 10 & 178.8359 & 178.9886 & 178.5279 & 178.6978 \\
\hline $10 \mathrm{a}$ & 135.0127 & 134.3492 & 134.9955 & 134.3676 \\
\hline 1 ' & 44.4818 & 44.4914 & 42.2666 & 42.2994 \\
\hline 2 , & 61.3373 & 61.3059 & 64.8547 & 64.8247 \\
\hline 3 ' & 74.4202 & 74.403 & 72.9174 & 72.9076 \\
\hline $4^{\prime}$ & 65.1159 & 65.0992 & 66.4062 & 66.4159 \\
\hline
\end{tabular}


Table S9. DP4+ evaluation of theoretical and experimental data.

\begin{tabular}{|c|c|c|c|c|c|c|c|}
\hline \multicolumn{2}{|c|}{ mPT 1PF91 } & \multicolumn{2}{|c|}{ Gas Phase } & \multicolumn{2}{|c|}{$G-31+G(d, p)$} & \multicolumn{2}{|c|}{ Unsca1ed Shifts } \\
\hline & & DP4+ & 제 $0.00 \%$ & $1100.00 \%$ & - & - & - \\
\hline Nuc1ei & sp2? & Experimenta & Isomer 1 & Isomer 2 & Isomer 3 & Isomer 4 & Isomer 5 \\
\hline C & $\mathrm{x}$ & 163.7 & 160.5398621 & 160.2620265 & & & \\
\hline C & $\mathrm{x}$ & 120.3 & $\mid 120.3142138$ & 118.5837643 & & & \\
\hline C & $\mathrm{x}$ & 163.5 & 160.8125557 & 162. 6728262 & & & \\
\hline C & $\mathrm{x}$ & 108.7 & 112. 6346307 & 112. 9064211 & & & \\
\hline C & $\mathrm{x}$ & 132.5 & 132. 8214605 & 132.5776603 & & & \\
\hline C & $\mathrm{x}$ & 108.6 & 107. 0622468 & 106. 6143717 & & & \\
\hline$C$ & $\mathrm{x}$ & 164.2 & 159. 9295736 & 159.6267933 & & & \\
\hline $\mathrm{C}$ & $\mathrm{x}$ & 108.1 & 106.0465074 & 105.8647553 & & & \\
\hline C & $\mathrm{x}$ & 165.0 & 163.7575551 & 163.5562064 & & & \\
\hline $\mathrm{C}$ & $\mathrm{x}$ & 109.7 & 110. 0633274 & 110. 2096178 & & & \\
\hline C & $\mathrm{x}$ & 188.7 & 185. 8747478 & 185.5480297 & & & \\
\hline C & $\mathrm{x}$ & 109.7 & 109. 4537468 & 108.2134657 & & & \\
\hline C & $\mathrm{x}$ & 181. 1 & 178. 6724081 & 178.8903172 & & & \\
\hline$C$ & $\mathrm{x}$ & 134.9 & 134. 4652224 & 134. 604996 & & & \\
\hline C & & 40.9 & 40. 10597791 & 44. 19514094 & & & \\
\hline $\mathrm{C}$ & & 59.8 & 73. 12093278 & 61. 7856186 & & & \\
\hline$C$ & & 70.7 & 82.75074736 & 74. 21046361 & & & \\
\hline C & & 64.6 & 66.28740854 & 65.27926161 & & & \\
\hline $\mathrm{H}$ & $\mathrm{x}$ & 6.52 & 6.749677844 & 6.735736756 & & & \\
\hline $\mathrm{H}$ & $\mathrm{x}$ & 7.04 & 7. 405677242 & 7. 372035501 & & & \\
\hline $\mathrm{H}$ & $\mathrm{x}$ & 7. 05 & 7. 822657093 & 7. 725699209 & & & \\
\hline $\mathrm{H}$ & & 3.67 & 3. 712615186 & 3. 988797975 & & & \\
\hline $\mathrm{H}$ & & 4. 18 & 4. 513520451 & 4. 002489734 & & & \\
\hline $\mathrm{H}$ & & 3.82 & 4. 388861573 & 4. 462171737 & & & \\
\hline $\mathrm{H}$ & & 3.58 & 4. 100830964 & 3. 590325079 & & & \\
\hline $\mathrm{H}$ & & 3.39 & 3. 521678398 & 3.819819843 & & & \\
\hline $\mathrm{H}$ & & 3.29 & |3. 266443038 & 3. 785442272 & & & \\
\hline
\end{tabular}


Table S10. Calculated ECD Data for (1'S,3'S)-1 in gas phase

\begin{tabular}{|c|c|c|c|c|c|c|}
\hline \multirow[b]{2}{*}{ State } & \multicolumn{2}{|l|}{$\mathrm{C1}$} & \multicolumn{2}{|l|}{$\mathrm{C2}$} & \multicolumn{2}{|l|}{$\mathbf{C 3}$} \\
\hline & $\begin{array}{l}\text { Excitation } \\
\text { energies(eV } \\
\text { ) }\end{array}$ & $\begin{array}{l}\text { Rotatory } \\
\text { Strengths* }\end{array}$ & $\begin{array}{l}\text { Excitation } \\
\text { energies }(\mathrm{eV} \\
\text { ) }\end{array}$ & $\begin{array}{l}\text { Rotatory } \\
\text { Strengths* }\end{array}$ & $\begin{array}{l}\text { Excitation } \\
\text { energies }(e V \\
)\end{array}$ & Rotatory Strengths* \\
\hline 1 & 3.1987 & 3.0096 & 3.1978 & 5.5896 & 3.1911 & -3.4631 \\
\hline 2 & 3.2304 & 6.1161 & 3.2241 & 3.3814 & 3.2539 & -14.8621 \\
\hline 3 & 3.7962 & 0.3166 & 3.7746 & 0.3872 & 3.8053 & 5.1482 \\
\hline 4 & 3.899 & -0.4958 & 3.8377 & -0.4624 & 3.8725 & 4.3657 \\
\hline 5 & 4.1533 & 1.1022 & 4.121 & 1.6316 & 4.1826 & -1.7167 \\
\hline 6 & 4.5254 & -8.2262 & 4.4481 & -8.356 & 4.5048 & 5.1646 \\
\hline 7 & 4.9133 & 1.95 & 4.931 & 1.0219 & 4.926 & 8.5843 \\
\hline 8 & 5.3385 & -2.0275 & 5.3545 & -1.7756 & 5.3441 & 6.916 \\
\hline 9 & 5.3955 & -3.8719 & 5.4105 & -3.869 & 5.3801 & -4.5631 \\
\hline 10 & 5.5603 & 4.8888 & 5.5205 & 4.4974 & 5.5541 & -9.692 \\
\hline 11 & 6.0377 & 10.9624 & 6.0303 & 13.7756 & 5.8556 & -3.2956 \\
\hline 12 & 6.1119 & 12.5037 & 6.1089 & 7.7503 & 5.9986 & -13.371 \\
\hline 13 & 6.1354 & -4.8488 & 6.1422 & 0.3117 & 6.122 & 47.1599 \\
\hline 14 & 6.2202 & 12.2508 & 6.2196 & 7.4146 & 6.1437 & -23.644 \\
\hline 15 & 6.2673 & 7.2489 & 6.2588 & 12.0844 & 6.2471 & 3.7479 \\
\hline 16 & 6.3217 & -0.1735 & 6.2902 & -0.6367 & 6.2941 & -6.3189 \\
\hline 17 & 6.3428 & -9.7386 & 6.3168 & -11.0913 & 6.3206 & -5.4995 \\
\hline 18 & 6.447 & -1.0773 & 6.4196 & -0.9299 & 6.3679 & 6.6845 \\
\hline 19 & 6.5087 & -0.8741 & 6.506 & -2.4762 & 6.4676 & -5.0244 \\
\hline 20 & 6.6899 & -3.3193 & 6.6755 & -1.906 & 6.5357 & 0.7054 \\
\hline 21 & 6.7432 & -0.476 & 6.7491 & -1.9103 & 6.5545 & 0.1435 \\
\hline 22 & 6.8754 & -2.5074 & 6.8742 & 1.6543 & 6.754 & -10.4415 \\
\hline 23 & 6.9035 & -2.6756 & 6.8962 & -3.8033 & 6.806 & -1.4118 \\
\hline 24 & 6.9185 & 16.252 & 6.9112 & 4.9713 & 6.8868 & 1.4818 \\
\hline 25 & 6.9323 & -18.766 & 6.9212 & -8.7653 & 6.9517 & -44.6319 \\
\hline 26 & 7.0299 & 1.1461 & 7.0029 & -4.443 & 6.9533 & 41.3044 \\
\hline 27 & 7.0597 & 5.8617 & 7.0497 & 11.0365 & 7.0504 & -15.4875 \\
\hline 28 & 7.1094 & 0.5314 & 7.1185 & -0.8999 & 7.1658 & 5.3088 \\
\hline 29 & 7.1711 & 3.2472 & 7.1826 & 2.3425 & 7.23 & -22.8747 \\
\hline 30 & 7.2684 & -7.0393 & 7.2822 & -1.6312 & 7.2693 & 7.2364 \\
\hline 31 & 7.2792 & 17.5363 & 7.2882 & 7.8115 & 7.2839 & 0.3918 \\
\hline 32 & 7.3893 & 0.4284 & 7.3903 & 14.7808 & 7.3208 & 8.0195 \\
\hline 33 & 7.4311 & -6.8275 & 7.4308 & -20.6732 & 7.3902 & 13.5882 \\
\hline 34 & 7.49 & -9.3163 & 7.4699 & -10.6195 & 7.438 & -19.4313 \\
\hline 35 & 7.5331 & 1.13 & 7.5561 & 3.0253 & 7.4431 & -1.9155 \\
\hline
\end{tabular}




\begin{tabular}{|l|l|l|l|l|l|l|}
\hline 36 & 7.5626 & -0.561 & 7.5737 & 9.8212 & 7.4681 & -6.1025 \\
\hline 37 & 7.6154 & -4.9853 & 7.5845 & -0.7666 & 7.4785 & 21.065 \\
\hline 38 & 7.6259 & -1.4985 & 7.6204 & -6.749 & 7.6591 & -0.6656 \\
\hline 39 & 7.6322 & -4.6566 & 7.6369 & -6.1422 & 7.7251 & 7.621 \\
\hline 40 & 7.6617 & 3.8601 & 7.6561 & 4.0284 & 7.7373 & -11.4947 \\
\hline 41 & 7.7211 & 0.4774 & 7.7119 & -1.1231 & 7.781 & 1.4906 \\
\hline 42 & 7.8263 & 7.7485 & 7.8365 & 5.9706 & 7.8183 & -4.0581 \\
\hline 43 & 7.8585 & 6.7717 & 7.8723 & 22.8016 & 7.8445 & 0.6516 \\
\hline 44 & 7.8731 & 6.2447 & 7.883 & -4.6585 & 7.8711 & 9.0937 \\
\hline 45 & 7.8871 & 5.7046 & 7.9043 & -5.4758 & 7.8895 & 13.2189 \\
\hline 46 & 7.9032 & -13.4732 & 7.941 & 4.86 & 7.9293 & -11.0029 \\
\hline 47 & 7.9317 & 8.8761 & 7.9821 & -3.3606 & 7.9366 & 19.5992 \\
\hline 48 & 8.0503 & -6.9372 & 8.0872 & 4.2823 & 7.9705 & -1.4457 \\
\hline 49 & 8.1093 & -8.5678 & 8.105 & -14.7906 & 8.0114 & 9.7046 \\
\hline 50 & 8.1421 & 8.7968 & 8.1491 & 12.3996 & 8.0692 & -1.3661 \\
\hline 51 & 8.146 & 11.6629 & 8.1706 & 0.3326 & 8.109 & 3.444 \\
\hline 52 & 8.1984 & -4.7049 & 8.2004 & 3.779 & 8.136 & 25.0581 \\
\hline 53 & 8.2174 & -0.797 & 8.212 & -2.6231 & 8.1927 & -34.6392 \\
\hline 54 & 8.2457 & 9.3773 & 8.2438 & 12.4196 & 8.2114 & -6.31 \\
\hline 55 & 8.2804 & 3.6095 & 8.2554 & -11.5551 & 8.2613 & 17.0183 \\
\hline 56 & 8.2926 & -1.57 & 8.2845 & 7.791 & 8.2702 & 1.3086 \\
\hline 57 & 8.3023 & 2.0313 & 8.318 & -5.3109 & 8.2813 & -4.1121 \\
\hline 58 & 8.3449 & 12.3739 & 8.3303 & 1.6687 & 8.3009 & 16.2074 \\
\hline 59 & 8.3665 & 8.6111 & 8.3444 & 19.9068 & 8.3121 & 6.8042 \\
\hline 60 & 8.398 & -0.2644 & 8.4022 & 0.4829 & 8.3237 & -19.6756 \\
\hline
\end{tabular}

* $\mathrm{R}$ (velocity) $10 * *-40$ erg-esu-cm 


\begin{tabular}{|c|c|c|}
\hline \multirow[b]{2}{*}{ State } & \multicolumn{2}{|l|}{$\mathbf{C 4}$} \\
\hline & Excitation energies $(\mathrm{eV})$ & Rotatory Strengths* \\
\hline 1 & 3.2075 & -4.7401 \\
\hline 2 & 3.2299 & -13.4932 \\
\hline 3 & 3.7777 & 8.8622 \\
\hline 4 & 3.8144 & 1.1368 \\
\hline 5 & 4.1502 & -2.2921 \\
\hline 6 & 4.4284 & 5.4014 \\
\hline 7 & 4.9438 & 8.2555 \\
\hline 8 & 5.3579 & 6.8296 \\
\hline 9 & 5.3922 & -5.9256 \\
\hline 10 & 5.5152 & -9.5246 \\
\hline 11 & 5.8194 & -2.5494 \\
\hline 12 & 5.9827 & -15.639 \\
\hline 13 & 6.1222 & 44.479 \\
\hline 14 & 6.1694 & -19.3618 \\
\hline 15 & 6.2418 & 5.549 \\
\hline 16 & 6.2674 & -2.0764 \\
\hline 17 & 6.2942 & -10.9912 \\
\hline 18 & 6.3383 & 6.4789 \\
\hline 19 & 6.4352 & -5.042 \\
\hline 20 & 6.5275 & -2.4169 \\
\hline 21 & 6.5367 & 2.1114 \\
\hline 22 & 6.7552 & -3.4693 \\
\hline 23 & 6.8036 & -3.8467 \\
\hline 24 & 6.9007 & -7.2828 \\
\hline 25 & 6.9139 & 0.583 \\
\hline 26 & 6.9671 & 1.0085 \\
\hline 27 & 7.0516 & -13.6198 \\
\hline 28 & 7.1772 & 5.9103 \\
\hline 29 & 7.2221 & -13.9362 \\
\hline 30 & 7.2661 & 4.8932 \\
\hline 31 & 7.2887 & 5.8052 \\
\hline 32 & 7.2996 & 0.393 \\
\hline 33 & 7.3699 & -23.9132 \\
\hline 34 & 7.3965 & 11.4323 \\
\hline 35 & 7.4423 & 9.6276 \\
\hline 36 & 7.4683 & 2.7183 \\
\hline 37 & 7.5845 & 3.1045 \\
\hline
\end{tabular}




\begin{tabular}{|c|c|c|}
\hline 38 & 7.6028 & -0.4192 \\
\hline 39 & 7.6848 & 0.1427 \\
\hline 40 & 7.7514 & -8.3922 \\
\hline 41 & 7.7574 & 0.5105 \\
\hline 42 & 7.823 & 3.9357 \\
\hline 43 & 7.8824 & 0.4527 \\
\hline 44 & 7.8942 & 0.7316 \\
\hline 45 & 7.9155 & 10.0361 \\
\hline 46 & 7.936 & 1.5539 \\
\hline 47 & 7.9683 & 8.8849 \\
\hline 48 & 7.9799 & 6.2798 \\
\hline 49 & 8.031 & 4.2381 \\
\hline 50 & 8.0831 & 0.3807 \\
\hline 51 & 8.1434 & 24.8574 \\
\hline 52 & 8.1875 & -8.7816 \\
\hline 53 & 8.206 & -16.9813 \\
\hline 54 & 8.2168 & -11.5426 \\
\hline 55 & 8.2508 & -17.0264 \\
\hline 56 & 8.256 & 21.6923 \\
\hline 57 & 8.28 & 5.671 \\
\hline 58 & 8.2908 & 10.8919 \\
\hline 59 & 8.3163 & -6.9875 \\
\hline 60 & 8.3361 & -0.5206 \\
\hline
\end{tabular}

* $\mathrm{R}$ (velocity) $10^{* *-40}$ erg-esu-cm 
A

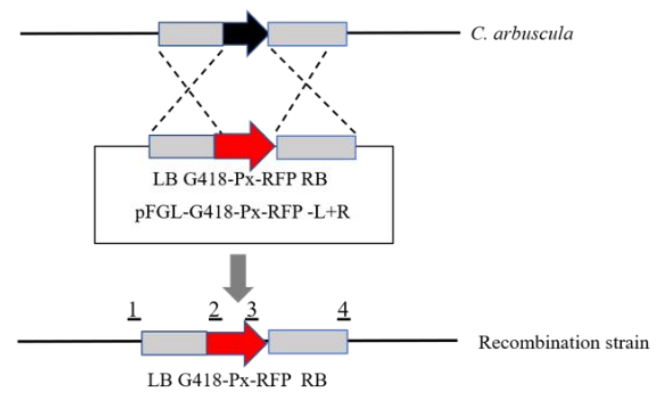

C

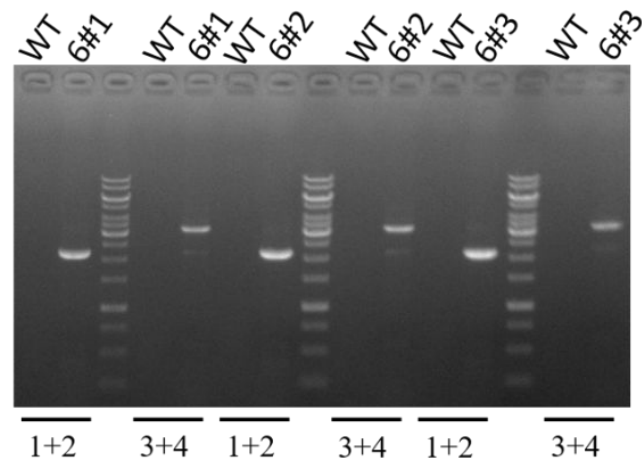

B

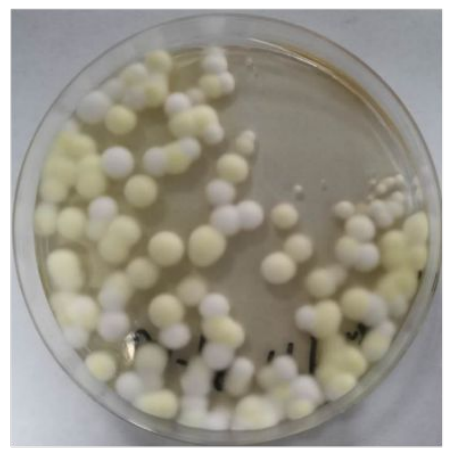

D

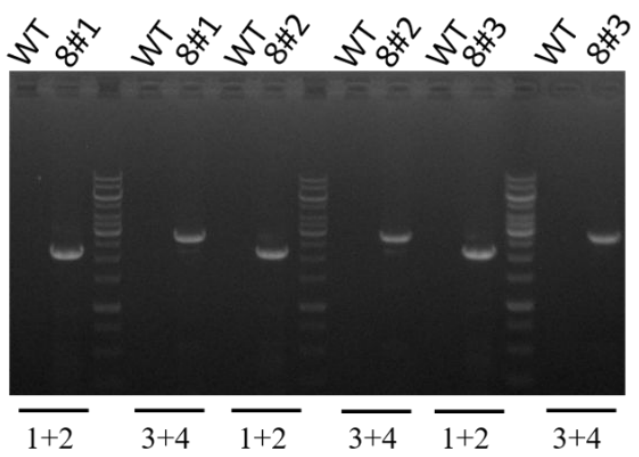

Figure S1. Insertion of the promoter expression cassettes on the aur $A$ locus.

A. Schematic diagram of ATMT-based gene disruption of the core area of aurA in C. arbuscula. neoR (G418 resistance gene) and $P x-R F P$ flanked by two homologous arms in T-DNA was used to replace the core area of aur $A$ on genome.

B. A plate with transformants for disruption of the core area of aurA gene in C. arbuscula. ATMT was carried out by introduction of the aurA disruption cassette in (A) into C. arbuscula wild type, and the plate was photographed after one week.

C and D. Confirmative PCR for the core area of aurA disruption. Recombination transformants with P6 and P8 for validation, respectively, with three transformants selected for each type. 

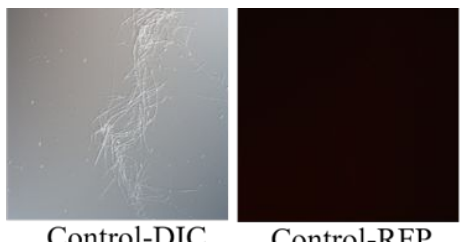

Control-RFP

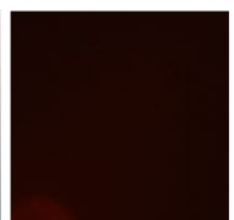

P3-RFP

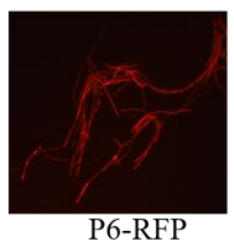

P6-DIC

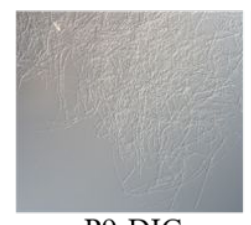

P9-DIC

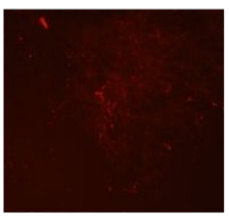

P9-RFP

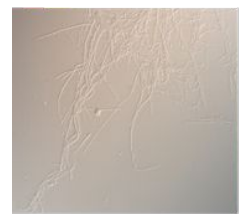

P12-DIC

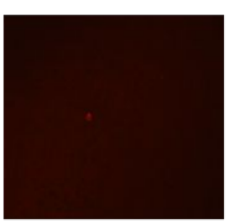

P12-RFP

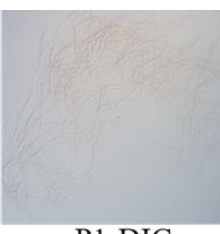

P1-DIC

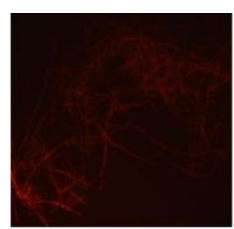

P1-RFP

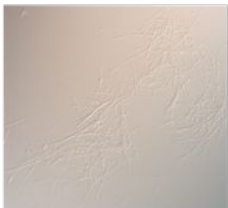

P4-DIC

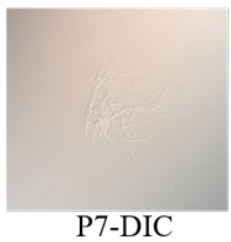

P7-DIC

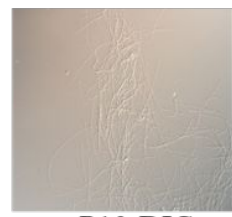

P10-DIC

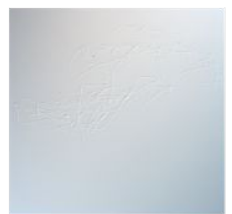

P13-DIC

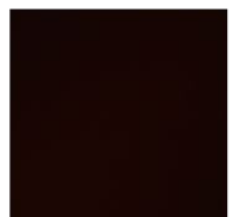

P4-RFP

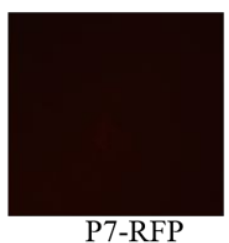

P7-RFP
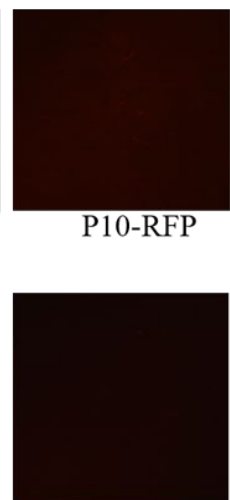

P13-RFP

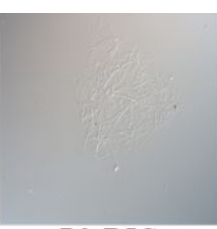

P2-DIC

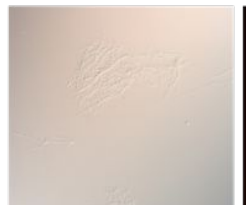

P5-DIC
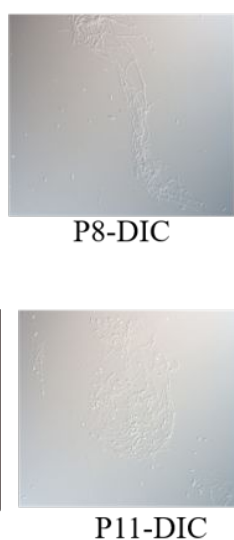

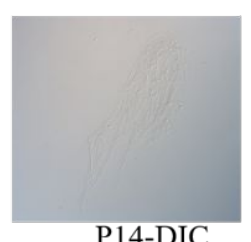

P14-DIC
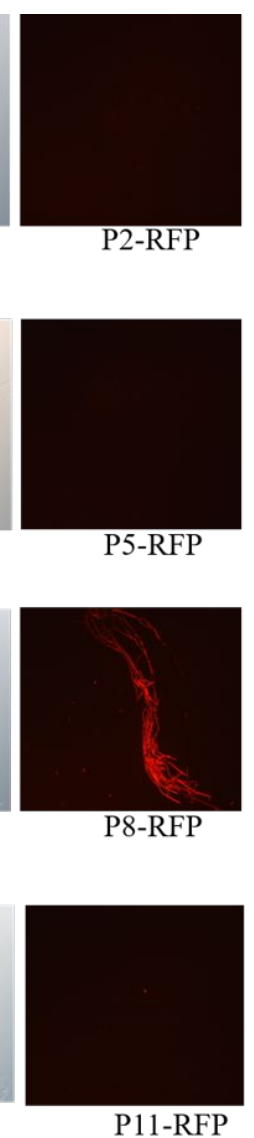

P2-RFP

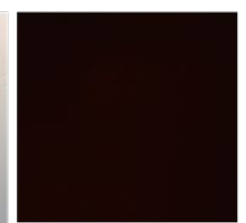

P5-RFP

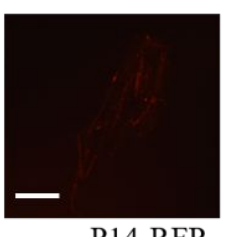

Figure S2. Expression of the red fluorescent protein (RFP) under 14 different promoters in $\triangle$ aurA. The observed mycelia were sampled from PDA for 6 days. $\triangle a u r A$ expressing RFP was observed in microscope with a red filter and cells without RFP were used as the negative control. Bar $=10 \mu \mathrm{m}$. 

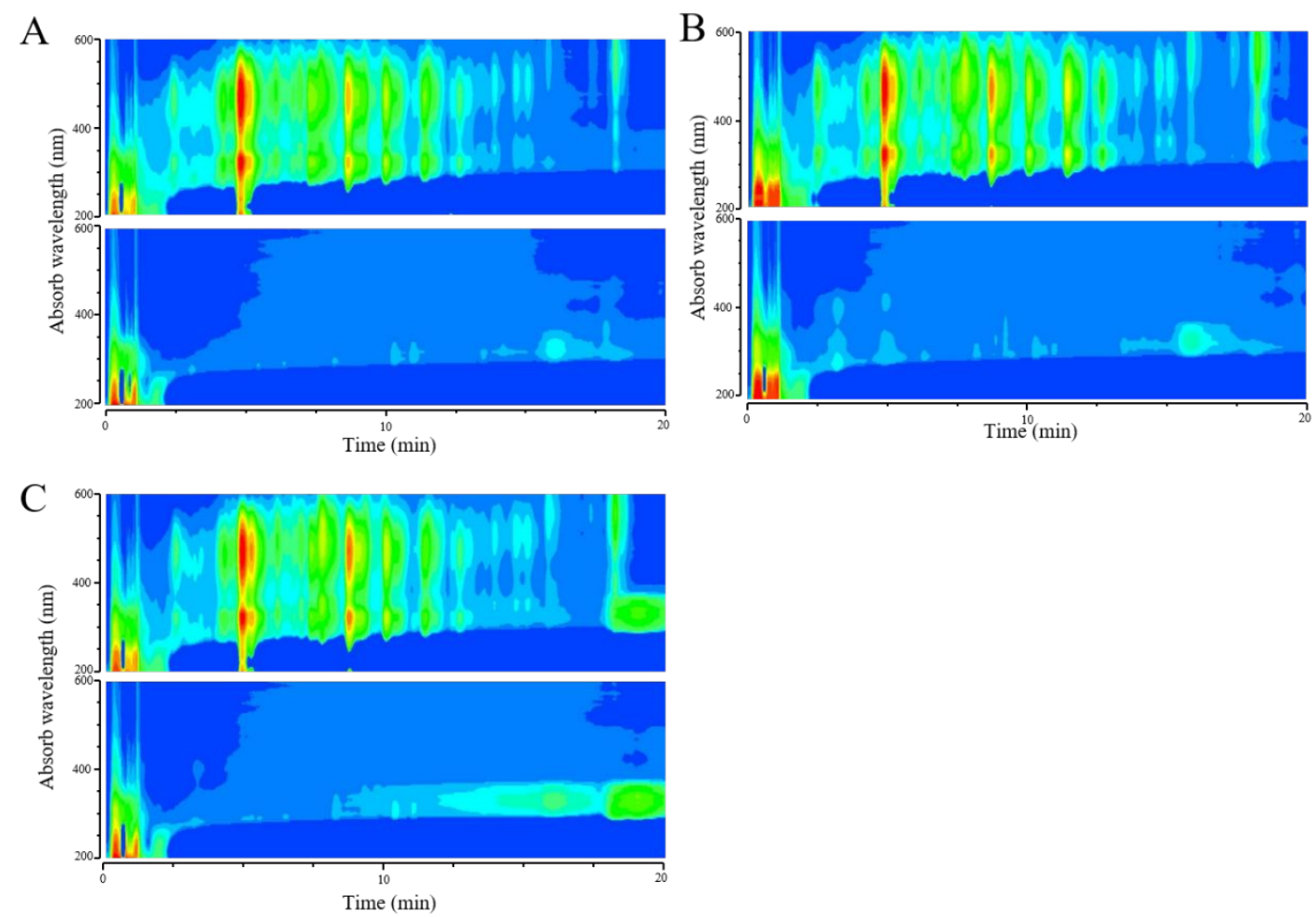

Figure S3. Metabolite profile analysis based on iso-absorbance plot.

C. arbuscula wild type (up) and C. arbuscula $\triangle a u r A$ (down) are inoculated about 6 days in different fermentation media, COBA (A), YGA (B) and YMEGA (C). The methanol extract of mycelium was analyzed on HPLC with wavelength from $200 \mathrm{~nm}$ to $600 \mathrm{~nm}$. 

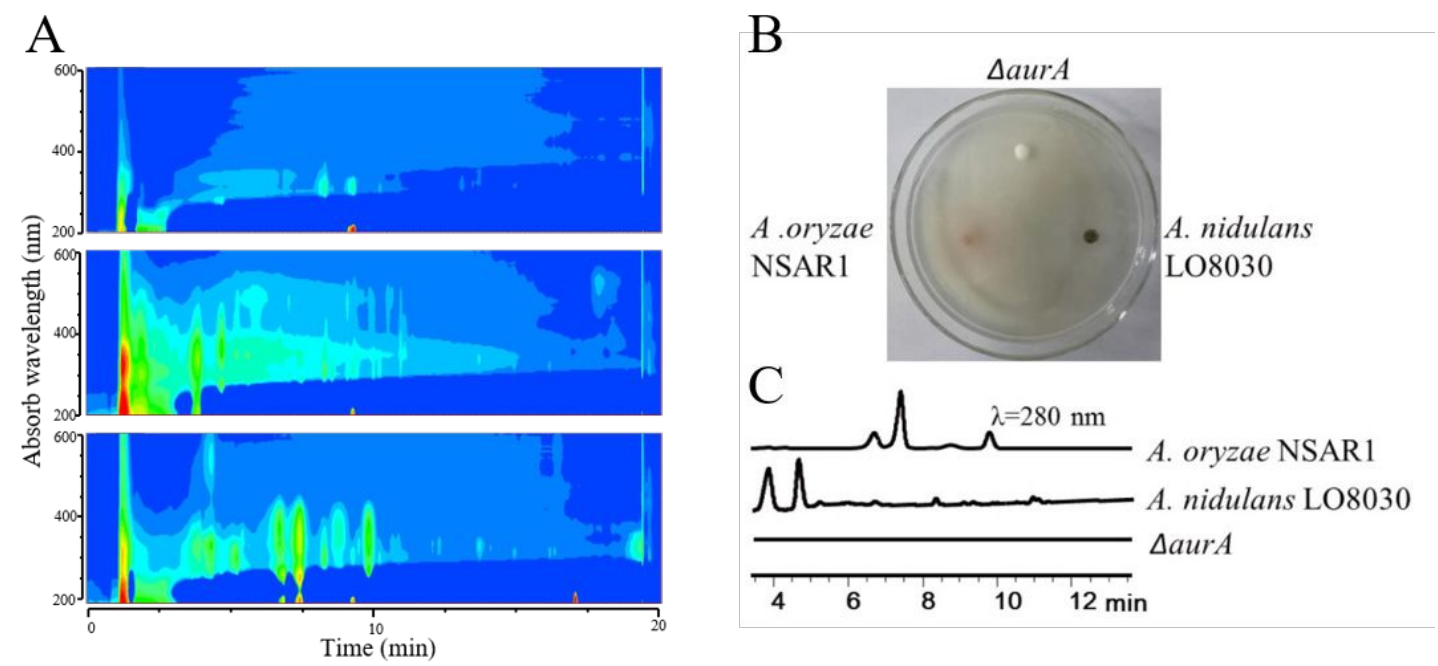

Figure S4. Metabolite profile analysis and HPLC analysis of three different hosts.

(A) Metabolite profile analysis based on iso-absorbance plot. C. arbuscula AaurA (down), A. nidulans LO8030 (middle) and A. oryzae NSAR1 (up) were inoculated on PDA and grown for about 6 days. The methanol extract of mycelia was analyzed on HPLC with wavelength from $200 \mathrm{~nm}$ to $600 \mathrm{~nm}$.

(B) Transformant identification via changes of colony colors. C. arbuscula $\triangle$ aurA was white, A. nidulans LO8030 was green and A. oryzae NSAR1 was red.

(C) HPLC analysis for confirmation of C. arbuscula $\triangle a u r A$, A. nidulans LO8030 and A. oryzae NSAR1. The methanol extract of C. arbuscula $\triangle a u r A, A$. nidulans LO8030 and A. oryzae NSAR1 was run on HPLC and detected at $\lambda=280 \mathrm{~nm}$.

For the culture of $C$. arbuscula $\triangle a u r A, A$. nidulans LO8030 and A. oryzae NSAR1, we inoculated an equal volume $(10 \mathrm{uL})$ of spores, the spore concentration is $10^{7} / \mathrm{mL}$, on the same volume size $(20 \mathrm{~mL})$ of PDA plate. After the cultivation, the plate (including culture medium and fungi) was extracted with $30 \mathrm{~mL}$ of methanol to obtain the crude material, and then $1 \mathrm{~mL}$ of each extract was filtered for HPLC test, and the injection volume was $30 \mathrm{uL}$. 


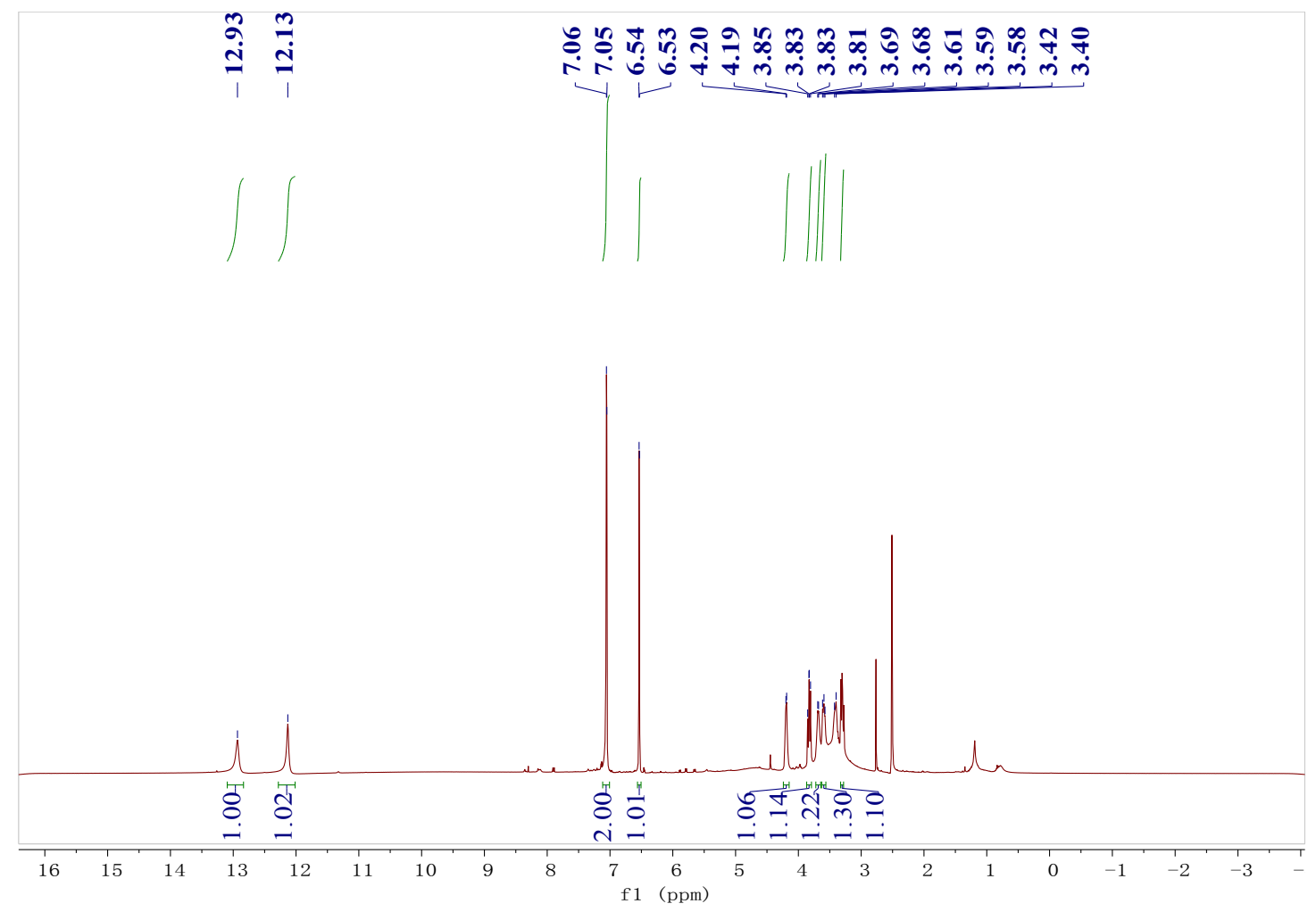

Figure S5. ${ }^{1} \mathrm{H}$ NMR spectrum of 1 in DMSO-d6 (500 MHz) 


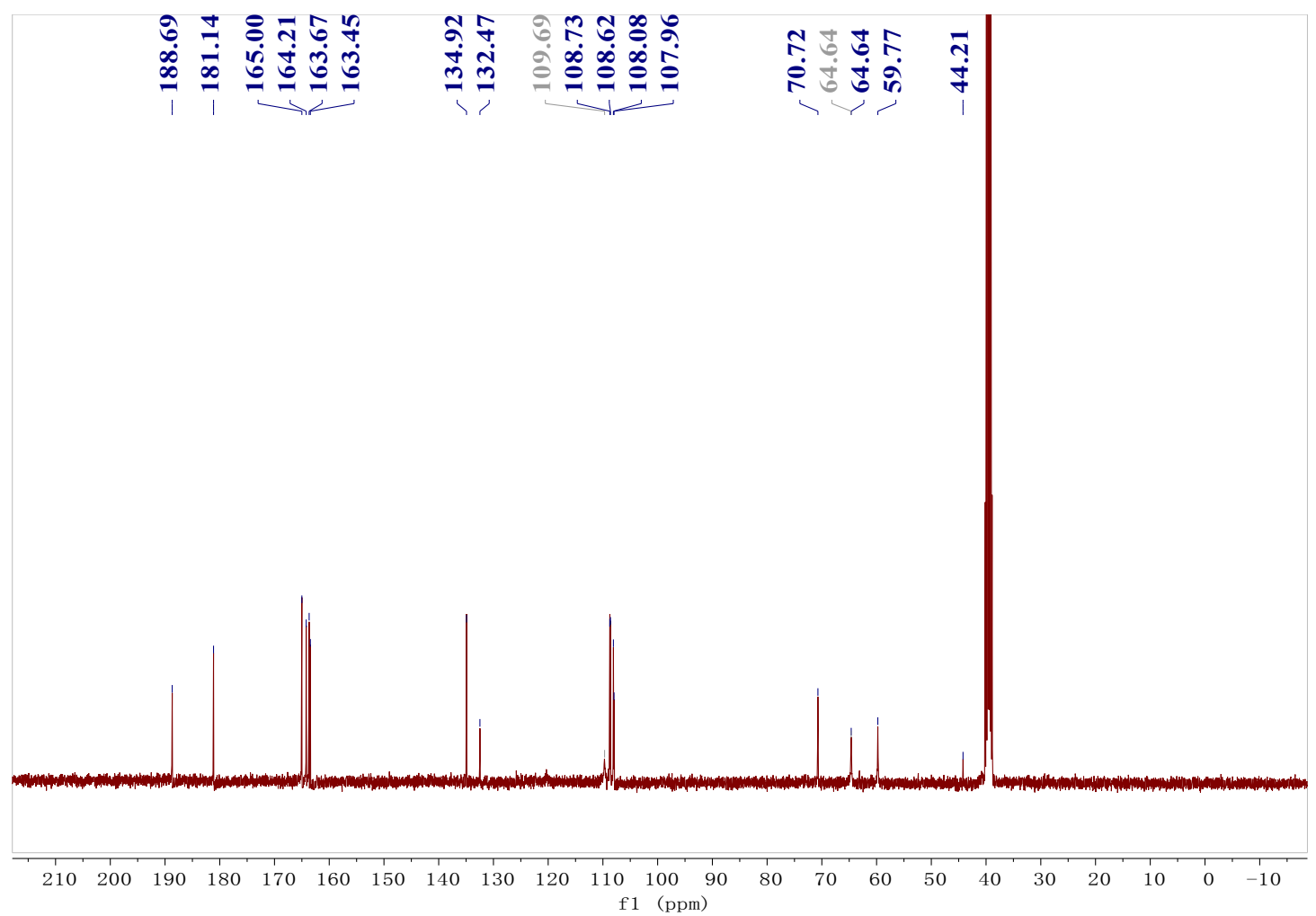

Figure S6. ${ }^{13} \mathrm{C}$ NMR spectrum of 1 in DMSO- $d 6$ 


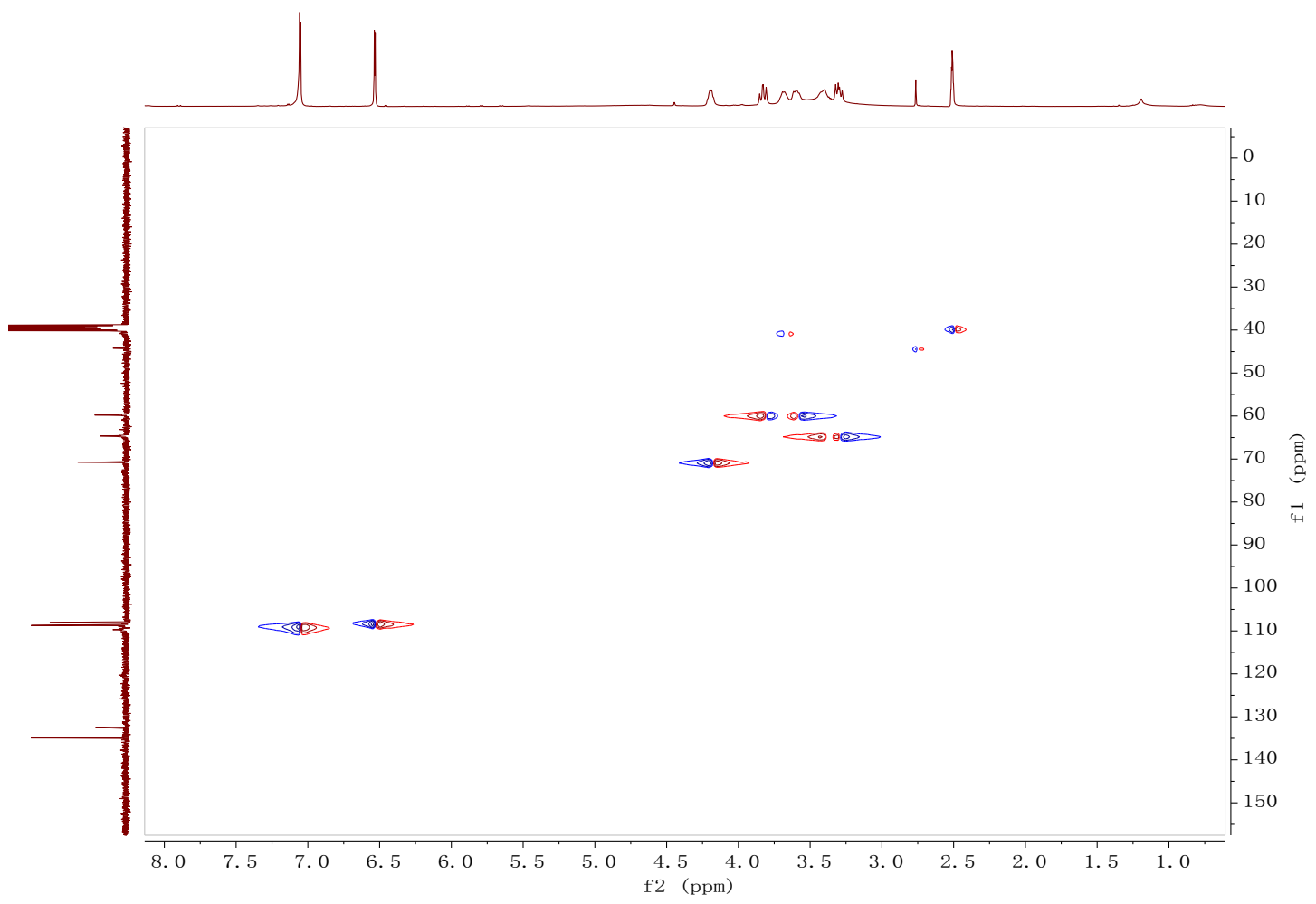

Figure S7. The HSQC spectrum of 1 in DMSO- $d 6$ 


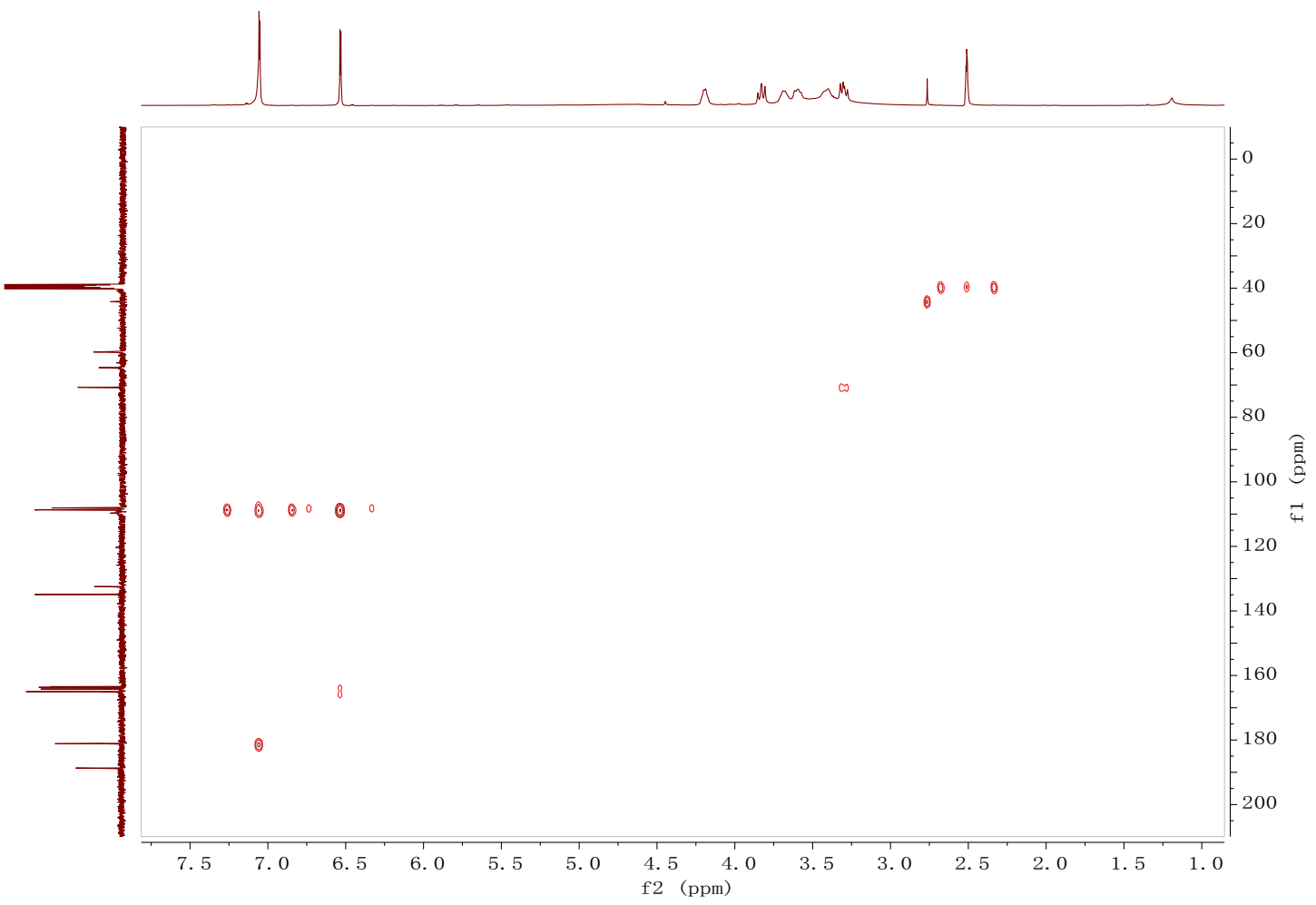

Figure S8. The HMBC spectrum of $\mathbf{1}$ in DMSO- $d 6$ 


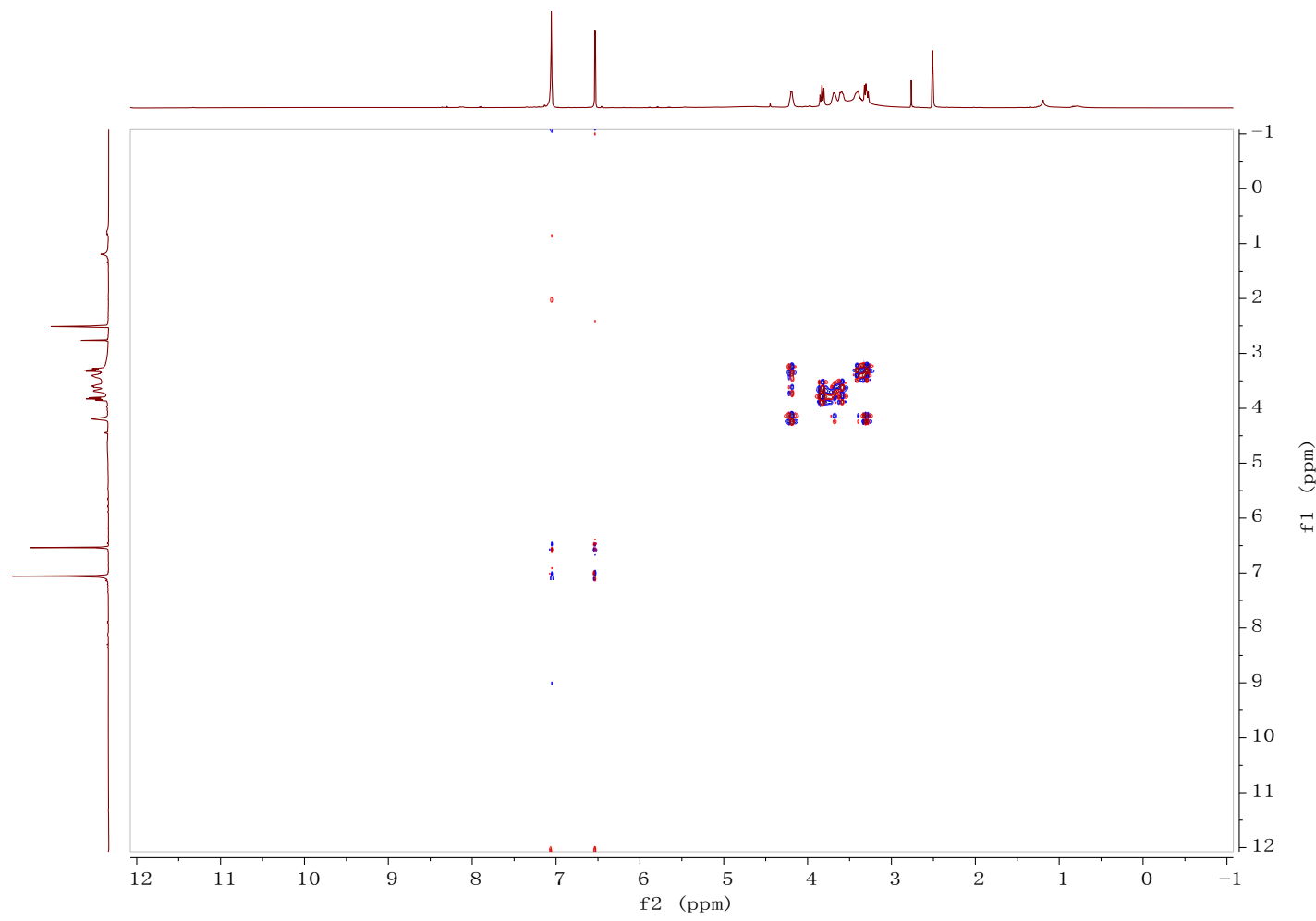

Figure S9. The COSY spectrum of $\mathbf{1}$ in DMSO-d6 


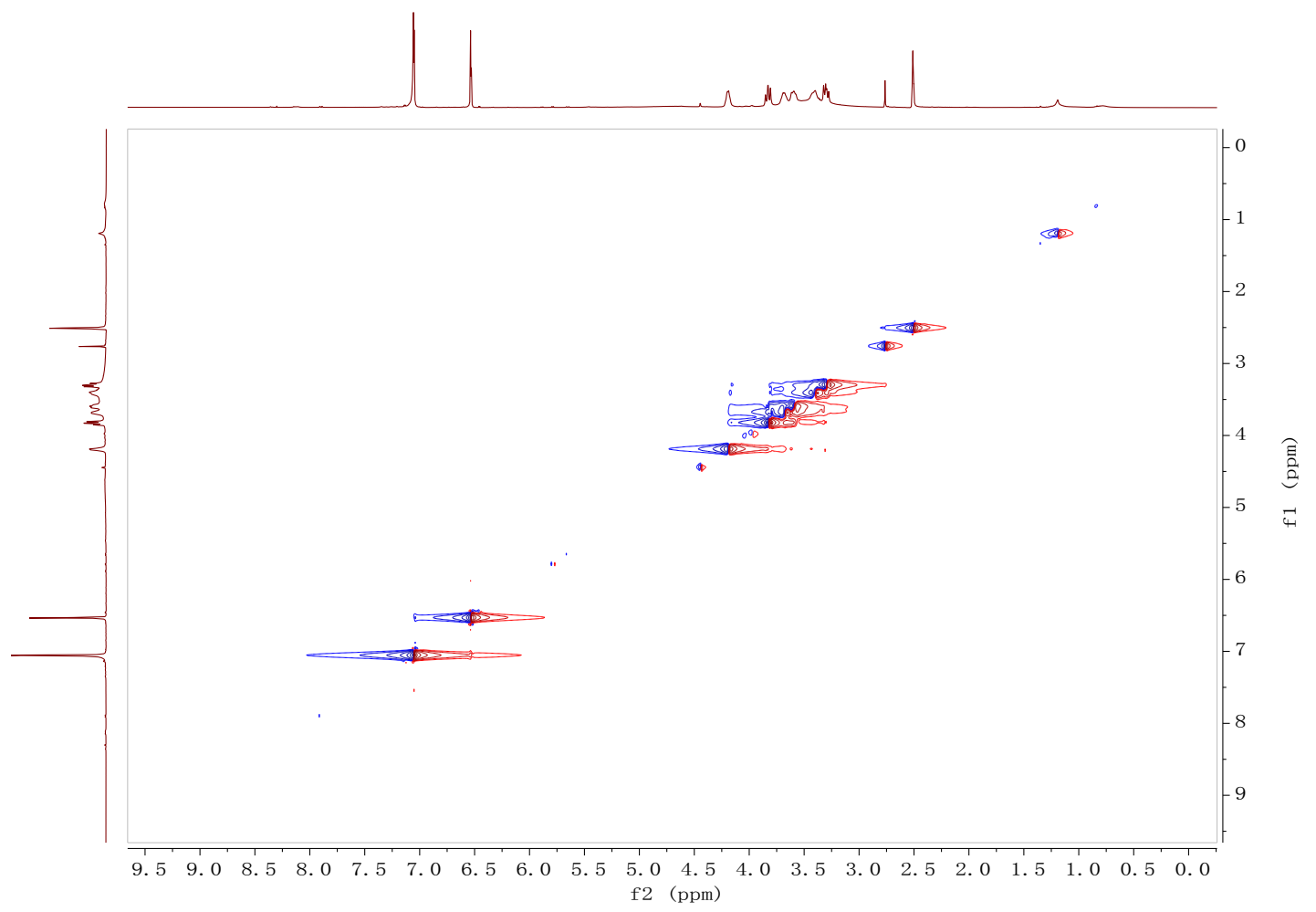

Figure S10. The NOESY spectrum of $\mathbf{1}$ in DMSO- $d 6$ 

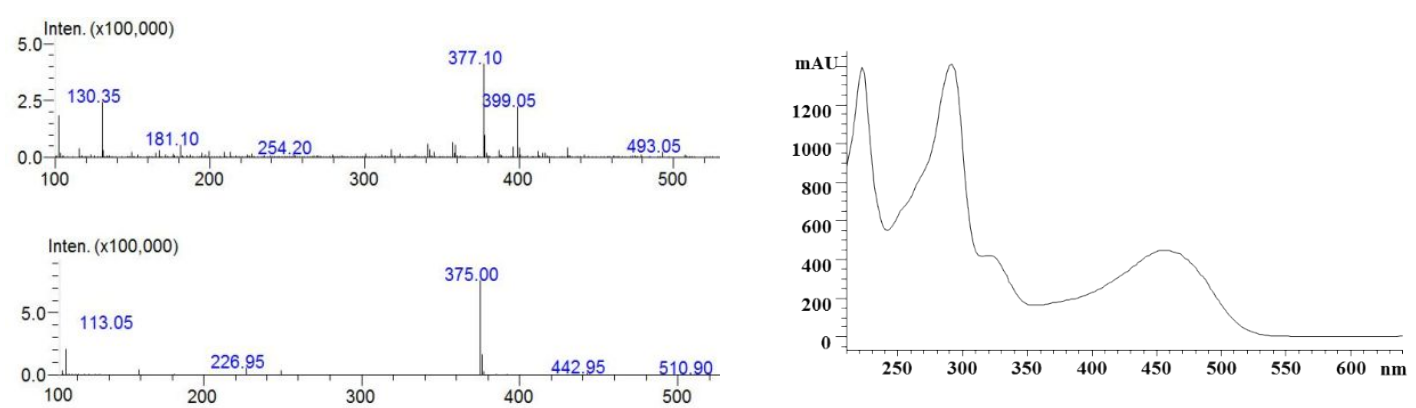

Figure S11. Determination of the structure of compound 1 .

Left panel: LC-MS analysis of compound $\mathbf{1}$ in positive and negative ion mode.

Right panel: UV-visible absorption of compound $\mathbf{1 .}$ 

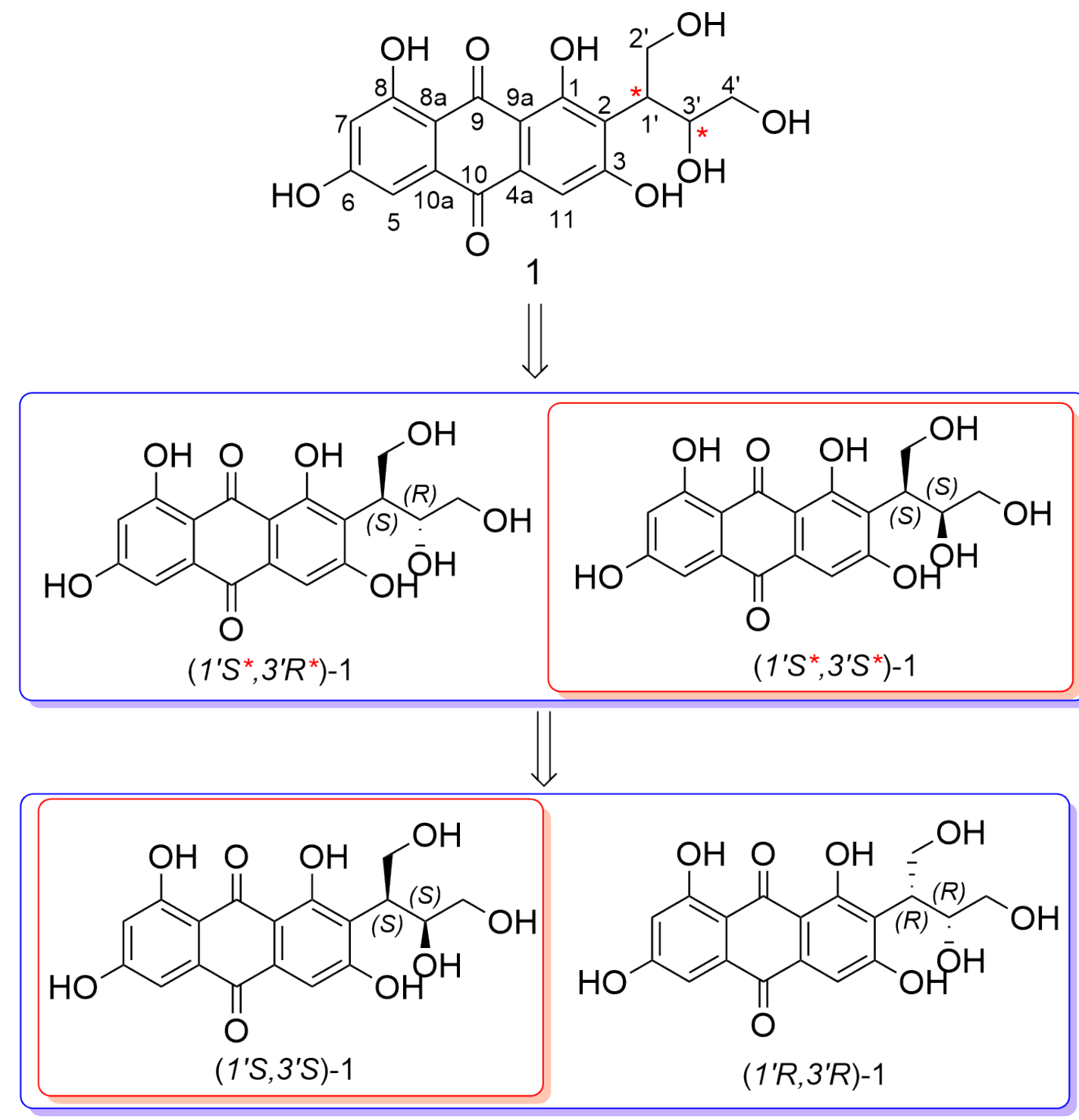

Figure S12. Determination of relative and absolute configuration of 1 


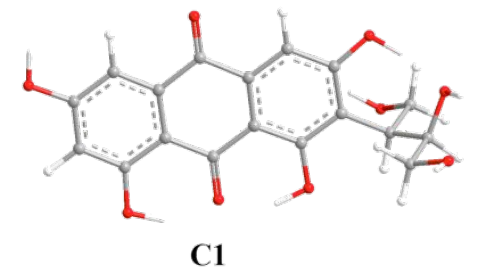

C1

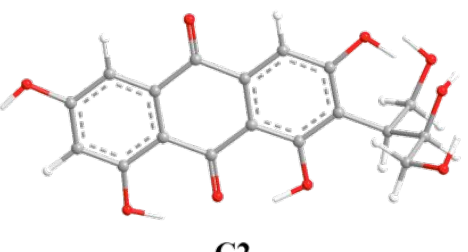

C2

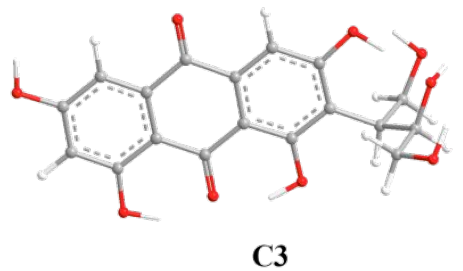

C3

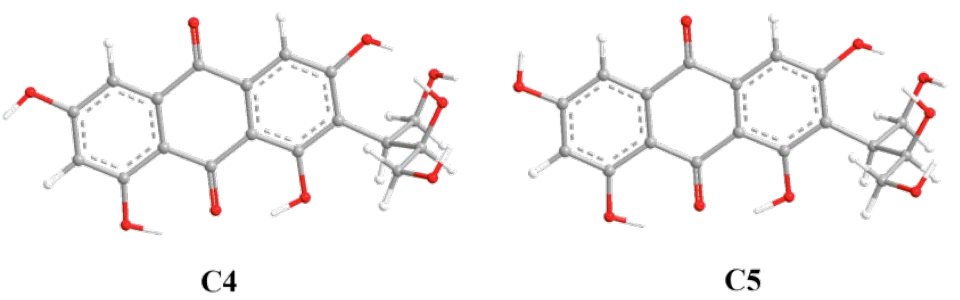

Figure S13. B3LYP/6-31G(d) optimized lowest energy conformers for $\left(1^{\prime} S, 3^{\prime} R\right)$-1. 

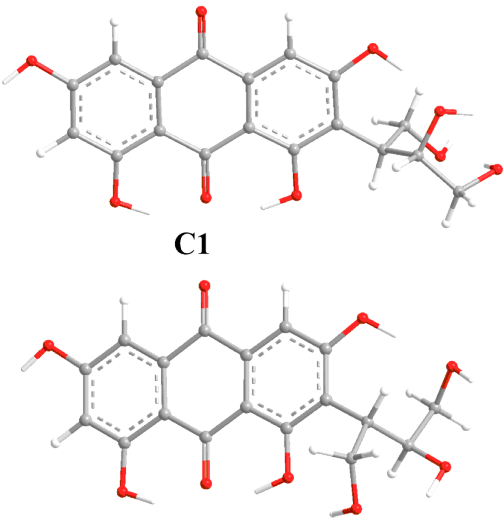

C3
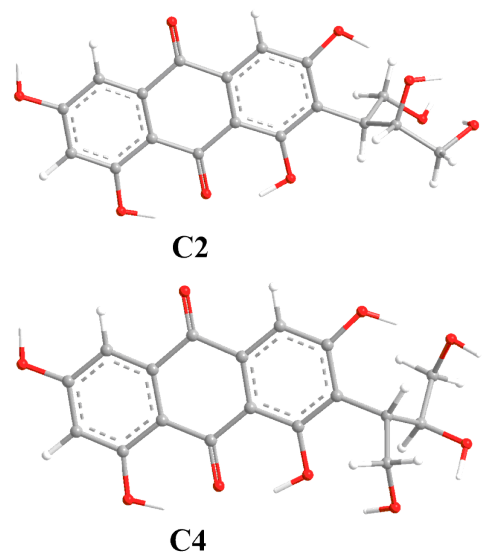

Figure S14. B3LYP/6-31G(d) optimized lowest energy conformers for (1'S,3'S) -1. 
A

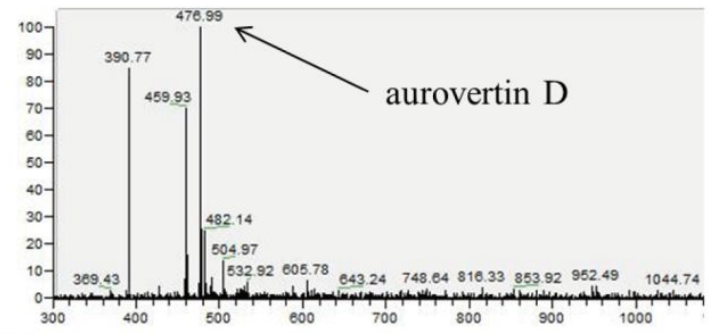

$\mathrm{C}$

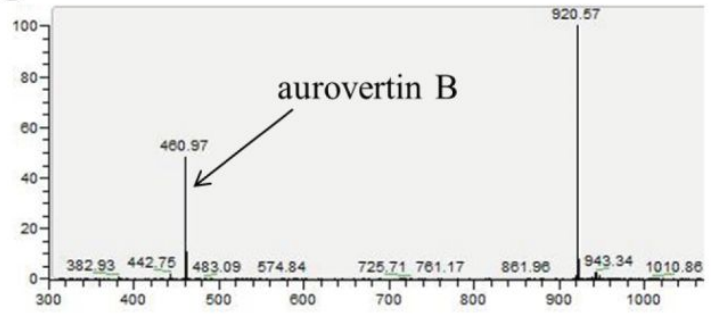

$\mathrm{E}$

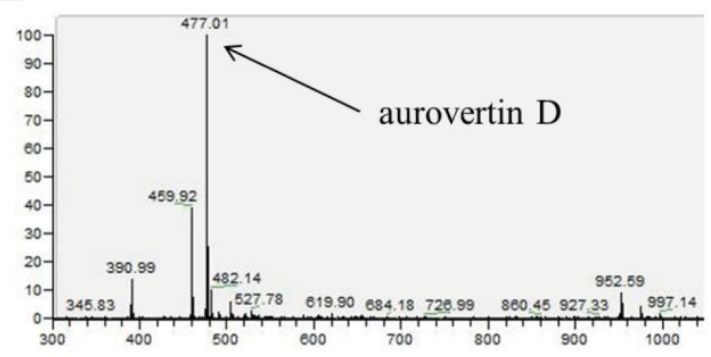

B

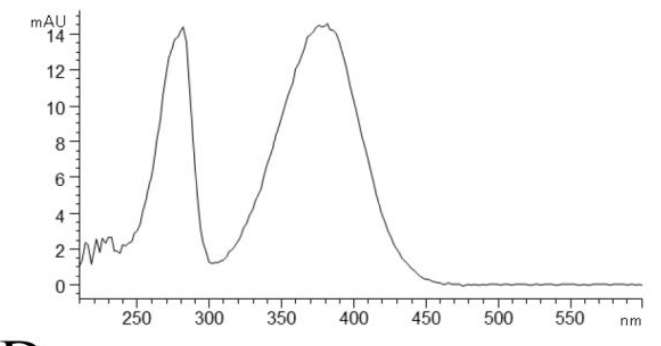

$\mathrm{D}$

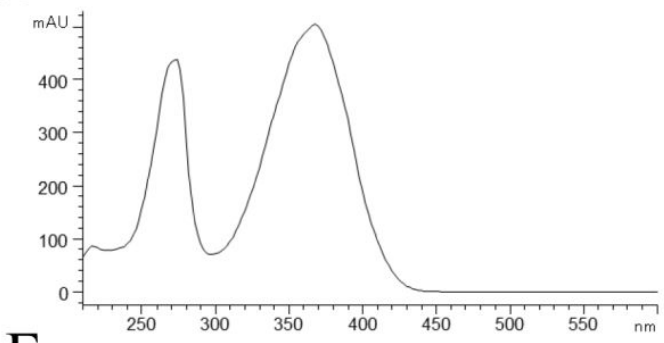

$\mathrm{F}$

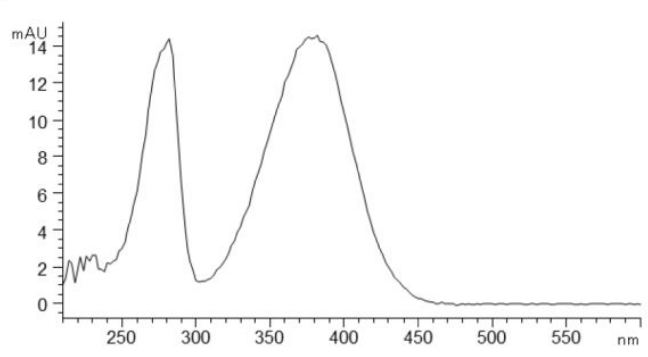

Figure S15. LC-MS analysis in positive ion mode and UV analysis of compound $\mathbf{2}$ and compound $\mathbf{3}$ (shown in Figure

$5)$.

(A) and (B) LC-MS analysis and UV analysis of compound 2 from $\triangle a u r A-m a v A$.

(C) and (D) LC-MS analysis and UV analysis of compound 3 from AaurA-mavA.

(E) and (F) LC-MS analysis and UV analysis of compound 3 from $\triangle a u r A-m r v A$. 

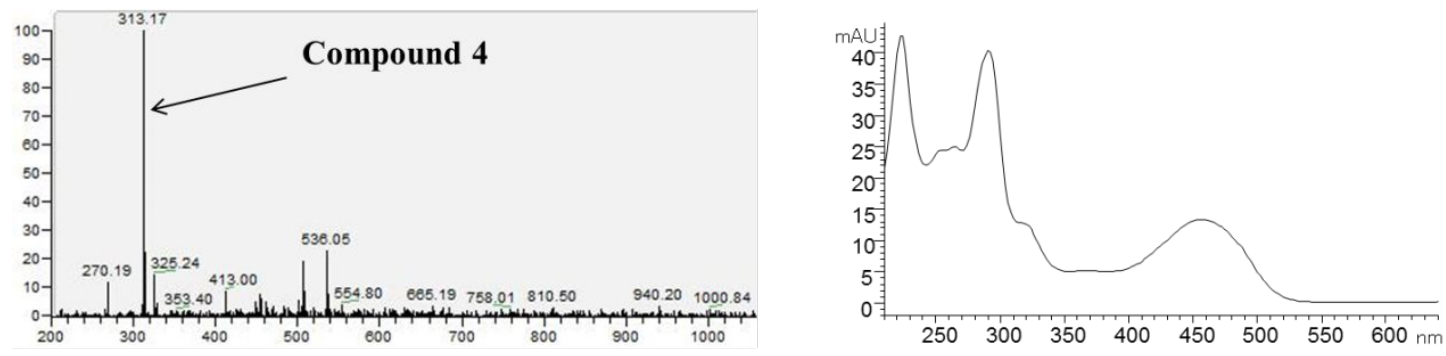

Figure S16. LC-MS analysis and UV analysis of compound 4.

Left panel: LC-MS analysis of compound 4 in negative ion mode. Right panel: UV-visible absorption of compound

4. 